Trauma Berufskrankh $2005 \cdot 7$ [Suppl 2]: S346-S353 DOI 10.1007/s10039-004-0947-9

Online publiziert: 11. November 2004

(c) Springer Medizin Verlag 2004

C. Knop · R. Schmid $\cdot$ M. Reinhold $\cdot$ M. Blauth

Universitätsklinik für Unfallchirurgie \& Sporttraumatologie, Medizinische Universität, Innsbruck, Österreich

\title{
Fixateur interne und Wirbelkörperersatz
}

\section{Indikation und Ergebnisse}

$\mathbf{H}_{\mathrm{u}}$ u et al. gaben 1996 die jährliche Inzidenz von Wirbelsäulenverletzungen mit 64 pro 100.000 Einwohner an. Am häufigsten waren die untere Halswirbelsäule $\left(\mathrm{C}_{4}-\right.$ C6) und der thorakolumbale Übergang betroffen. Etwa die Hälfte aller Verletzungen der gesamten Brust- und Lendenwirbelsäule fielen auf T11-L2 [11, 41, 62, 86]. Der Altersdurchschnitt der Patienten lag in großen Kollektiven übereinstimmend zwischen 30 und 40 Jahren [31, 41, 62].

Im deutschsprachigen Raum hat sich die von Magerl et al. 1994 [61] vorgestellte Klassifikation zunehmend durchgesetzt. Die Grundlage bildeten das morphologisch-mechanistische 2-Säulen-Konzept nach Whitesides, das aus einer auf Druck beanspruchten vorderen und einer auf Zug belasteten, hinteren Säule besteht. Die AO-Klassifikation nach Magerl berücksichtigt eine zunehmende biomechanische Instabilität der Verletzung, eine höhere Rate neurologischer Zusatzverletzungen und damit die schlechtere Prognose mit der Zunahme der Schwere der Verletzung von A nach C $[41,61,62]$.

Das von Böhler 1934 formulierte Behandlungsziel ist bis heute gültig:

\section{() „Wir wollen nicht nur, daß die Lähmung verschwindet, sondern daß der Verletzte auch seine frühere normale Form, Beweglichkeit und Kraft wiedererlangt."}

Diesem Ziel fügten Blauth et al. 1998 [11] hinzu,

\section{(7) „daß wir einen schmerzfreien Patienten wünschen, der allen Tätigkeiten uneingeschränkt und möglichst so wie vor dem Unfall nachgehen kann."}

\section{Stabilität und Instabilität}

Um dieses unumstrittene Behandlungsziel $\mathrm{zu}$ erreichen, erlangte der Begriff der segmentalen Stabilität oder Instabilität einer Läsion zunehmende Bedeutung [11]. Die unterschiedliche biomechanische Stabilität verschiedener Verletzungsmuster ist aus Versuchen an humanen Präparaten im Belastungssimulator in vitro bekannt. Problematisch ist jedoch die Analyse der akuten traumatischen Instabilität in vivo: Hier kann das Ausmaß der Instabilität nur anhand von bildgebenden Verfahren, d. h. aufgrund einer statischen Momentaufnahme abgeschätzt werden. Zur apparativen Standarddiagnostik gehören dabei Röntgenaufnahmen in 2 Ebenen (a.-p. und seitlich) und eine Computertomographie, möglichst mit biplanaren Rekonstruktionen. Blauth [10] teilte die Verletzungen in 3 abgestufte Gruppen unterschiedlicher Stabilität ein:

- Als stabil gelten Verletzungen, bei denen keine wesentliche weitere Veränderung der Stellung in Ruhe oder bei Belastung zu erwarten ist. Hierzu zählten z. B. Kompressionsverletzungen der Gruppen A 1 und A 2 nach Magerl [61], bei denen keine Verletzung der Bandscheibe anzunehmen ist.
- Bereits ab Gruppe A 2.3 (Kneifzangenbruch) liegt eine geringgradige Instabilität vor. Zu diesem Instabilitätsgrad zählen auch die Kompressionsverletzungen mit Beteiligung der Hinterwand des Wirbelkörpers-die sog. Berstungsbrüche (Gruppe A 3). Die Verletzungen dieser Gruppen weisen eine zusätzliche Verletzung mindestens einer angrenzenden Bandscheibe auf. Auch bei funktioneller Behandlung (ohne Stabilisierung) besteht bei diesem Instabilitätsausmaß jedoch keine Gefahr einer sekundären neurologischen Schädigung [11]. Die Grauzone der geringgradigen Instabilität wird dadurch gekennzeichnet, dass diese Verletzungen in der aktuellen Literatur als stabil [64] oder als instabil [62] eingeschätzt wurden.

- Distraktions- (Typ B) und Rotationsverletzungen (Typ C) werden als hochgradig instabil bezeichnet, da hier eine Verletzung der vorderen und hinteren Säule vorliegt. Ohne Stabilisierung besteht die Gefahr einer sekundären neurologischen Komplikation. Blauth et al. [11] stuften bereits komplette Berstungsbrüche (A 3.3) als hochgradig instabil ein. Dafür sprechen die Ergebnisse biomechanischer Untersuchungen und das mögliche Auftreten sekundärer neurologischer Komplikationen bei konservativer Behandlung. Andere Autoren bezeichneten diesen Frakturtyp ebenfalls als instabil, als instabil mit Angabe zusätzlicher Bedingungen oder sogar als grundsätzlich stabil. 
Die akute traumatische Instabilität führt - je nach Ausmaß - bei fehlender operativer Stabilisierung zu einer zunehmenden kyphotischen Fehlstellung mit Fehlbelastung der kleinen Wirbelgelenke und des Bewegungssegmentes. Liegt eine asymmetrische Verletzung vor, beispielsweise durch seitliche Kompression eines Wirbelkörpers, kann eine skoliotische Verformung hinzukommen. Verletzte Bänder heilen dabei unter Ausbildung einer Narbe, die nur eingeschränkt funktionsfähig ist und in geringerem Maße Zugkräften widersteht. Knöcherne Verletzungen der Wirbelanteile heilen in der Regel belastungsstabil unter Konsolidierung der Spongiosa, wobei sich nur ausnahmsweise Kallus nachweisen lässt.

Eine Verletzung der Bandscheibe führt zur Vernarbung, mit der die Funktion verloren geht. Im Verlauf kann es durch zunehmende Höhenminderung des Zwischenwirbelraumes und Kyphosierung im Bewegungssegment zur knöchernen Überbrückung und damit stabilen Ausheilung in der Fehlstellung kommen. Tritt nach der Bandscheibenverletzung keine knöcherne Fusion ein, resultiert eine bleibende (Mikro)instabilität, die zu erheblichen statischen Folgen für das Achsorgan und zu chronischen Schmerzen führen kann.

\section{Behandlungsmethoden und Ergebnisse}

Da Verletzungen der Brust- und Lendenwirbelsäule selten sind, bestehen nur begrenzte Möglichkeiten, über größere Patientengruppen zu berichten. Daher sind die im Schrifttum geschilderten Behandlungsergebnisse nach unterschiedlichen Therapieformen meistens von eingeschränkter Aussagekraft.

Die Kollektive der überwiegend retrospektiven Sammelstudien waren oft äußerst inhomogen, und es wurden unterschiedliche Regionen und Verletzungen nicht getrennt betrachtet. Oft fehlte die differenzierte Betrachtung bei inkompletten und kompletten Lähmungen, bei unterschiedlich langen Zeiträumen zwischen Unfall und Behandlungsbeginn und nicht zuletzt bei verschiedenen Behandlungsmethoden. Dabei wurden Ergebnisse uneinheitlich erhoben und unterschiedlichste, fast ausnahmslos nicht validierte Scores verwendet, so- dass subjektive und funktionelle Ergebnisse kaum vergleichbar waren [11].

Stabile Verletzungen ohne neurologisches Defizit werden auch heute noch fast ausnahmslos konservativ behandelt. Dabei wird in Anlehnung an die Empfehlungen von Magnus und Böhler entweder funktionell ohne äußere Ruhigstellung, mit äußerer Ruhigstellung durch Gipsmieder oder Orthese oder mit äußerer Ruhigstellung nach geschlossener Reposition behandelt.

\section{() Wenn nichtoperativ behandelt wird, dann sollte die funktionel- le Therapie ohne Reposition oder äußere Ruhigstellung ge- wählt werden.}

Ein Vorteil durch Gipsmieder- oder Orthesenbehandlung konnte gegenüber der rein funktionellen Therapie ohne äußere Ruhigstellung nicht bewiesen werden. Korrekturverluste und/oder Sinterungen von verletzten Wirbelkörpern scheinen in gleichem Maße einzutreten [39]. Es kam auch unter konservativer Behandlung von stabilen Verletzungen zu einem geringen, zusätzlichen Korrekturverlust gegenüber der Fehlstellung zum Unfallzeitpunkt. Wurde die Fehlstellung zunächst reponiert und anschließend ruhiggestellt, so folgte ein entsprechend höherer Korrekturverlust im Sinne einer Rekyphosierung, sodass im Endergebnis gegenüber der funktionellen Behandlung kein Vorteil erzielt werden konnte [72].

\section{Operativ - Nichtoperativ}

Die Grenze zwischen operativer und nichtoperativer Behandlung bei den inkompletten Berstungsbrüchen ist nicht sicher $\mathrm{zu}$ ziehen.

Die Empfehlungen zur Behandlung geringgradig instabiler Verletzungen ohne neurologisches Defizit reichen von der oben genannten konservativen bis hin $\mathrm{zu}$ aufwändigsten operativen Therapieverfahren. Nach konservativer Behandlung waren z. T. erhebliche Korrekturverluste gegenüber dem Ausgangsbefund nach dem Unfall zu verzeichnen. Das röntgenologische Ergebnis fiel in vergleichenden Untersuchungen nach operativer Therapie besser aus $[3,4,20]$.
Trauma Berufskrankh 2005 - 7 [Suppl 2]:

S346-S353

DOI 10.1007/s10039-004-0947-9

c) Springer Medizin Verlag 2004

\section{Knop · R. Schmid · M. Reinhold}

M. Blauth

\section{Fixateur interne und Wirbelkörperersatz. Indikation und Ergebnisse}

\section{Zusammenfassung}

Die Autoren schildern die Grundlagen aktueller Behandlungskonzepte für die Verletzungen der Brust- und Lendenwirbelsäule. Unter Bezugnahme auf biomechanische Konzepte und die Klassifikation der Verletzungen werden Erfahrungen und Ergebnisse geschildert, die bis heute erzielt und veröffentlicht wurden. Die Vor- und Nachteile von operativen und nichtoperativen Behandlungskonzepten werden dargestellt. Außerdem schildern die Autoren die verschiedenen Operationsmethoden, die heute zur Anwendung kommen. Erste eigene Ergebnisse mit dem Wirbelkörperersatzimplantat Synex werden vorgestellt.

\section{Schlüsselwörter}

Wirbelkörperersatzimplantat · Synex · Wirbelsäulenverletzungen · Biomechanische Stabilität · Behandlungskonzepte

\section{Internal fixator and vertebral body replacement. Indication and results}

\section{Abstract}

The authors explain the basic principles of current treatment concepts for injuries of the thoracic and lumbar spine. Referring to biomechanical concepts and classification of the injuries, they report on experiences gathered and results obtained to date which have been published. The advantages and disadvantages of surgical and nonsurgical treatment concepts are discussed. The authors also describe the various surgical techniques currently in use. Initial results with the Synex implant for vertebral body replacement are presented.

\section{Keywords}

Implant for vertebral body replacement . Synex - Spinal injuries - Biomechanical stability $\cdot$ Treatment concepts 


\section{Frakturen des thorakolumbalen Übergangs}
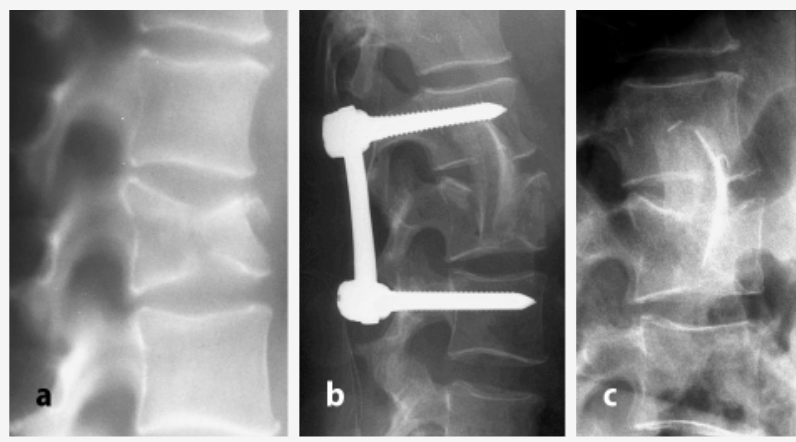

Abb. $1 \Delta$ 50-jährige Patientin mit Kneifzangenbruch (A2.3) von L1, konventionelle Tomographie (a); kombiniertes Vorgehen mit dorsaler Reposition und Stabilisierung mit bisegmentalem Fixateur interne sowie ventraler monosegmentaler Fusion mit Beckenkammspan (b); Ausheilungsergebnis 2 Jahre postoperativ mit eingeheiltem Span in unveränderter Stellung (c)

Ob die operierten Patienten ebenfalls ein besseres funktionelles Ergebnis und weniger Beschwerden aufweisen, konnte bis heute nicht abschließend geklärt werden. Nach sehr unterschiedlichen konservativen Behandlungsmethoden und nur begrenzter Vergleichbarkeit wurden „sehr gute“ Ergebnisse bei 0-71\% der Patienten erzielt, wobei 6 der 8 Publikationen eine Rate von $24-43 \%$ „sehr guter“ Ergebnisse aufwiesen [2, 14, 34, 36, 60, 66, 79, 90]. Nach verschiedenen Operationsmethoden wurden „sehr gute“ Ergebnisse bei $36-77 \%$ gefunden $[1,18,24,34,38,63]$. Die Rate „schlechter“ Ergebnisse scheint mit O-10\% nach operativer Therapie $[1,18,24$, $34,38,63$ ] niedriger zu sein als nach konservativer: Hier wurden $0-36 \%$,schlechte" Ergebnisse beobachtet [2, 14, 34, 36, 6o, $66,79,90]$.

\section{Operationsindikation}

Eine Operationsindikation liegt bei mehr als $15^{\circ}$ segmentaler kyphotischer Fehlstellung vor.

Mit jeder Operation ist ein zusätzliches Weichteiltrauma verbunden, das selbst auch für Schmerzen verantwortlich gemacht werden kann. Hinzu kommen je nach Verfahren - unterschiedlich hohe Komplikationsraten. Damit verknüpft ist die Frage nach einer akzeptablen Fehlstellung: Ist die vollständige Wiederherstellung der physiologischen Wirbelsäulenkrümmung erforderlich oder können posttraumatische Kyphosen innerhalb gewisser Grenzen toleriert werden? Welches
Ausmaß einer segmentalen Fehlstellung führt zu messbar stärkeren Beschwerden?

Als Grenze für die konservative Behandlung am thorakolumbalen Übergang wurde eine Verminderung der Wirbelkörpervorderkantenhöhe auf 40-50\% genannt. Andere gaben eine Kyphose von $30^{\circ}$ oder eine Höhenminderung des Wirbelkörpers um 1/3 an. Trotzdem beruhten diese Empfehlungen auf individueller Erfahrung und Empirie, denn eine Mehrzahl von Autoren fand keinen Zusammenhang zwischen der segmentalen Fehlstellung und subjektiven Beschwerden. Einige Autoren konnten eine Korrelation zwischen den genannten subjektiven und objektiven Parametern nachweisen $[58,78]$. Trojan [82] fand vermehrte Beschwerden $\mathrm{ab} 20^{\circ}$ Kyphose und Day und Kokan [19] zufolge verschlechterte eine Wirbelkörperkompression von mehr als 50\% die Prognose. Tropiano et al. [83] fanden ein signifikant schlechteres Outcome bei einer segmentalen Fehlstellung von $>15^{\circ}$.

\section{"Versteckte", ligamentäre Distraktionsverletzung}

Für die Diagnostik bzw. den Ausschluss der „versteckten“, ligamentären Distraktionsverletzung („hidden $\mathrm{B}_{1}$ “) ist Folgendes zu beachten.

Die Unterscheidung zwischen „reiner Typ-A-Verletzung und einer ligamentären Flexions-Distraktions-Verletzung mit Wirbelkörperfraktur (B1) ist hier von besonderer Bedeutung: In Rückenlage des Patienten kann die Verletzung leicht übersehen werden [56]. Die röntgenologischen Zeichen für eine Distraktionsverletzung, wie beispielsweise ein erhöhter Dornfortsatzabstand in der a.-p.-Aufnahme, können durch „Spontanreposition" unsichtbar bleiben, und es kommt bei funktioneller Behandlung zu einer erheblichen Kyphosierung durch die höhere Instabilität der tatsächlichen Verletzung. Im Zweifelsfall sollte bei funktioneller Behandlung einer angenommenen A-Verletzung frühzeitig nach Mobilisation des Patienten eine Röntgenkontrolle im Stehen erfolgen.

Es besteht heute weitgehende Übereinstimmung darin, dass hochgradig instabile Verletzungen (Typ B und C nach der Magerl-Klassifikation [61]) und diejenigen mit neurologischer Zusatzverletzung operiert werden sollten $[1,18,21,23,31,33$, $38,50,51,59,65,75,81]$. Offen bleibt dabei die Frage, wie operiert werden soll, und es werden unterschiedliche Zugangswege sowie Stabilisierungs- und Fusionstechniken angegeben. Retrospektive Nachuntersuchungsergebnisse nach operativer Behandlung wurden oft nur anhand kleiner Kollektive erhoben, die inhomogen waren oder eine Auswahl darstellten. Systematische und prospektive Erhebungen zu Vorund Nachteilen der einzelnen Verfahren konnten bis heute nicht vorgelegt werden.

\section{Dorsale Operationstechnik}

In Europa kommen heute für die dorsale Reposition und Stabilisierung von Verletzungen fast ausnahmslos winkelstabile Fixateursysteme zum Einsatz. Langstreckige Implantate - wie beispielsweise das Harrington-Instrumentarium oder LuqueStäbe - haben aufgrund hoher Komplikationsraten und geringerer Effektivität keine Bedeutung mehr. Die Komplikationsraten bei Einsatz des Fixateur interne erscheinen deutlich niedriger - auch im Vergleich zu ventralen Verfahren [42].

Der winkelstabile Fixateur interne bietet gute Möglichkeiten zur Reposition aller Fehlstellungen, kann jedoch allein einen postoperativen Korrekturverlust nicht verhindern. Als zusätzliche Techniken der Fusion von dorsal werden die interlaminäre Spondylodese sowie eine transpedikuläre intra- oder interkorporelle Spongiosaplastik nach Daniaux [18] verwendet. Mit dieser Technik konnte jedoch ein postoperati- 
ver Korrekturverlust nicht verhindert werden. Das Ziel einer sicheren interkorporellen Fusion konnte mit dieser Methode nicht erreicht werden $[9,17,37,43,44,57$, 70, 73, 85, 87, 88, 88].

Eine gute Alternative zur ventralen Rekonstruktion über einen gesonderten $\mathrm{Zu}$ gang scheint bei monosegmentalen Verletzungen und gleichzeitig notwendiger Dekompression die interkorporelle Fusion mit autogenem Beckenspan in PLIF-Technik zu sein (Kathrein et al., Jahrestagung DGU, Berlin 2002). Mittelfristige Ergebnisse liegen hier noch nicht vor.

Im eigenen Vorgehen wird die dorsale Reposition und Stabilisierung unabhängig von der Art der ventralen Rekonstruktion mit einer interlaminären Fusion kombiniert. Seltene Ausnahmen sind knöcherne Distraktionsverletzungen ohne Wirbelkörperfraktur, die lediglich eine Zuggurtung für die knöcherne Ausheilung benötigen.

Eine gute Alternative zur ventralten Rekonstruktion über einen gesonderten $\mathrm{Zu}$ gang scheint bei monosegmentalen Verletzungen und gleichzeitig notwendiger Dekompression die interkorporelle Fusion mit autogenem Beckenspan in PLIF-Technik zu sein (Kathrein et al. Jahrestagung DGU, Berlin 2002) Mittelfristige Ergebnisse liegen hier noch nicht vor.

Im eigenen Vorgehen wird die dorsale Reposition und Stabilisierung unabhängig von der Art der ventralen Rekonstruktion mit einer interlaminären Fusion kombiniert. Seltene ausnahmen sind knöcherne Distraktionsverletzungen ohne Wirbelkörperfraktur, die lediglich eine Zuggurtung für die knöcherne Ausheilung benötigen.

\section{Ventrale Operationstechnik}

Das rein ventrale Vorgehen bietet den Vorteil, dass die Läsion der vorderen Säule also des Wirbelkörpers - direkt mit einer druckfesten Abstützung durch einen Knochenspan und/oder ein Implantat angegangen werden kann $[6,27,38,51,92]$. Als Vorteil wurde außerdem die bessere Möglichkeit einer vollständigen Dekompression genannt. Doch auch bei dieser Technik wurden Korrekturverluste beobachtet und weitere Nachteile des Verfahrens bekannt: Der ventrale Zugang sei bei Pati- enten mit begleitender Lungenkontusion oder polytraumatisierten kontraindiziert, eine vollständige Reposition kaum möglich, verhakte Luxationen könnten irreponibel bleiben, skoliotische Fehlstellungen träten aufgrund des asymmetrischen Vorgehens in der Frontalebene häufiger ein, und die ventrale Stabilisierung sei zudem biomechanisch weniger stabil [11, 40].

Bis heute fehlt ein Implantat für die ventrale Stabilisierung, das eine vergleichbare und vollständige instrumentelle Reposition wie der dorsale Fixateur interne ermöglicht [42]. Es wurden für diese Technik erhebliche Komplikationsraten und zusätzliche verfahrensspezifische Probleme angegeben: Dazu gehörten die höhere Morbidität des ventralen Zugangs und der Spanentnahme am Beckenkamm sowie Spanpseudarthrosen und -frakturen.

\section{Kombinierte dorsoventrale Operationstechnik}

Bei der kombinierten dorsoventralen Operation wird die Verletzung zunächst mit einem Fixateur dorsal reponiert und stabilisiert, was auch als Notfalleingriff möglich ist. Danach wird ein- oder zweizeitig über einen separaten Zugang die ventrale $\mathrm{Ab}$ stützung und Fusion herbeigeführt. Dieses Vorgehen soll die Vorteile einer bestmöglichen Instrumentierung von dorsal mit der optimalen und sicheren ventralen Fusion vereinen $[11,12]$.

Doch auch bei diesem Verfahren wurden Nachteile deutlich: Die aufwändigere Operationstechnik war mit signifikant längerer Operationszeit und höherem Blutverlust verbunden [42]. Mehrere Autoren wiesen auf eine erhebliche Morbidität und Komplikationsmöglichkeiten durch die Spanentnahme am Beckenkamm hin, und es fanden sich ebenfalls Hinweise auf verfahrensspezifische Komplikationen wie Einheilungsstörungen oder Frakturen des Spanes $[5,15,35,52,76,77,80,89,91]$.

Die Technik des ventralen Zugangs und der ventralen Rekonstruktion konnte in den vergangenen 5 Jahren nachhaltig verbessert werden. So wurde die Spantechnik modifiziert und die Züricher Spantechnik mit „Nut-und-Feder-Prinzip“ verlassen (- Abb. 1). Heute werden die angrenzenden, intakten Endplatten nicht mehr "geopfert“, sondern lediglich sorgfältig , ange- frischt" und zur Abstützung des Spanes genutzt (• Abb. 2). Die Entnahmemorbidität am Beckenkamm kann - besonders für die bisegmentale Fusion - durch die Verwendung von Wirbelkörperersatzimplantaten wie beispielsweise Synex (• Abb. 3) vermieden werden $[54,55]$.

Neben dem Vorteil, dass auf die Entnahme eines Beckenkammspanes für die ventrale Abstützung verzichtet werden kann, ist die endoskopische Applikation des Implantates heute möglich [47]. Die Überlegenheit des Implantates (Synex ${ }^{\mathrm{Tm}}$, Synthes) wurde in biomechanischen Versuchen im Vergleich zum Harms-Korb bewiesen [45, 46]. Die besten röntgenologischen Ergebnisse mit dem geringsten Korrekturverlust wurden nach Rekonstruktion der ventralen Säule über einen vorderen Zugang angegeben [38, 40, 54, 67, 84].

Die Zugangsmorbidität war bisher ein wesentlicher Nachteil der vorderen $\mathrm{Zu}$ gänge. Nach ersten Berichten über die endoskopische Wirbelsäulenchirurgie 1993 und 1994 wurde das thorakoskopische Vorgehen von Beisse und Potulski für die Frakturversorgung am thorakolumbalen Übergang entwickelt und standardisiert. Bei besserer Visualisierung konnte die $\mathrm{Zu}$ gangsmorbidität signifikant gesenkt werden $[7,8,16,28,29,32,53,69]$.

\section{Vorgehen bei neurologischem Defizit}

In der prospektiven Multicenter-Studie der Arbeitsgemeinschaft „Wirbelsäule“ konnte zwischen dem Ausmaß eines neurologischen Defizites im Frankel-/ASIA-Score und der Einengung des Spinalkanals nach dem Unfall ein signifikanter Zusammenhang nachgewiesen werden [42]. Weder für inkomplette noch für komplette Querschnittssysndrome konnte jedoch bis jetzt der Nachweis geführt werden, dass eine operative Dekompression gegenüber einer konservativen Behandlung allein überlegen wäre. Boerger et al. [13] bestätigten diese Tatsache in einem aktuellen ReviewArtikel: Die Autoren kommen zu dem Schluss, dass eine Operationsindikation allein aufgrund eines neurologischen Defizites nicht gegeben ist. Die Aussagekraft des Artikels wird jedoch durch Sammlung von teilweise sehr kleinen Patientenkollektiven und die sehr unterschiedlichen und 


\section{Frakturen des thorakolumbalen Übergangs}
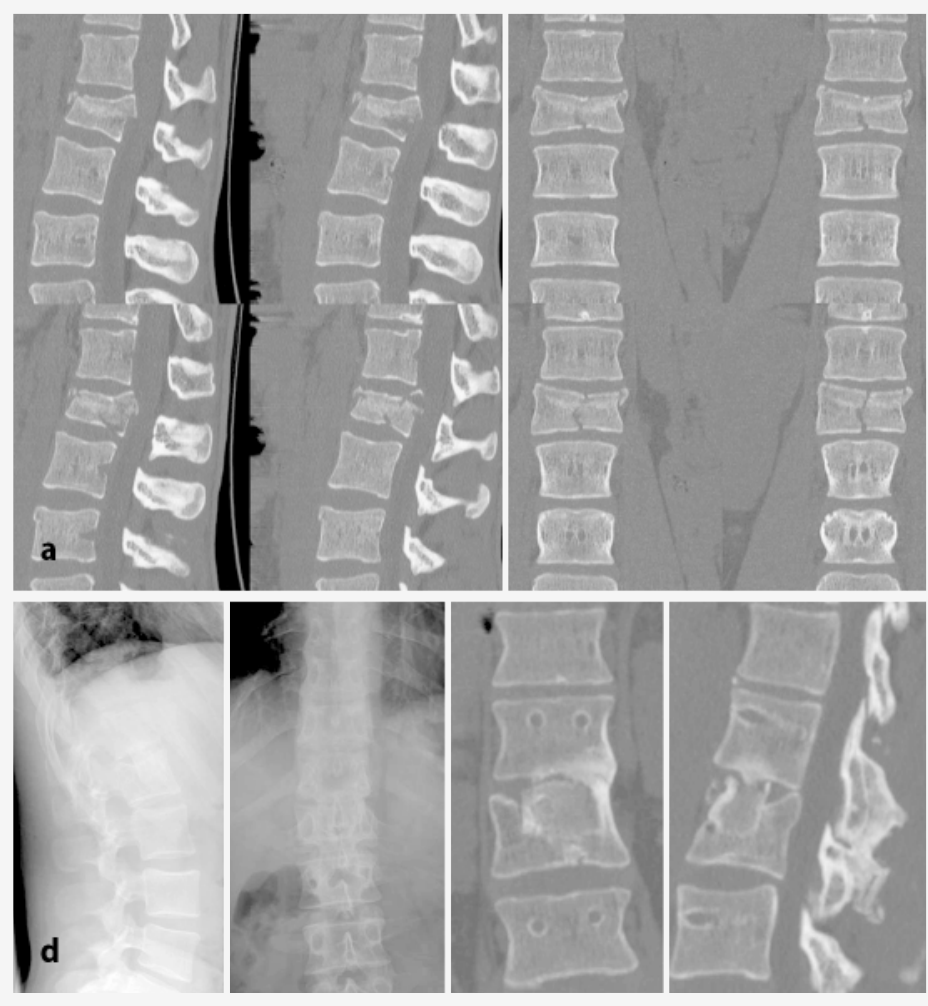
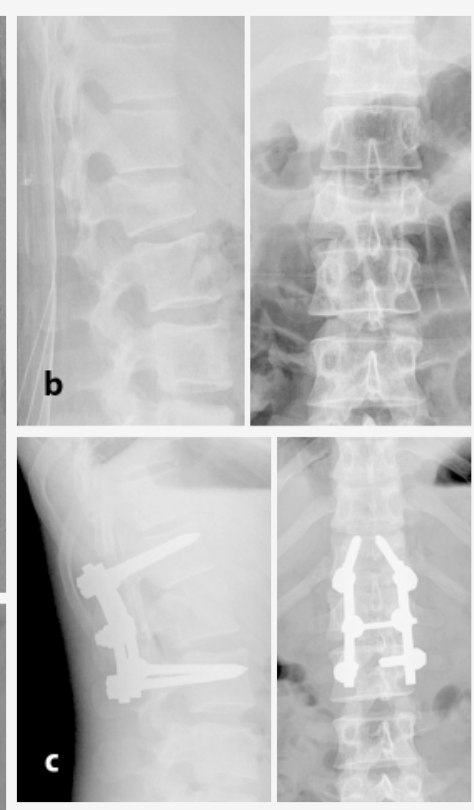

c

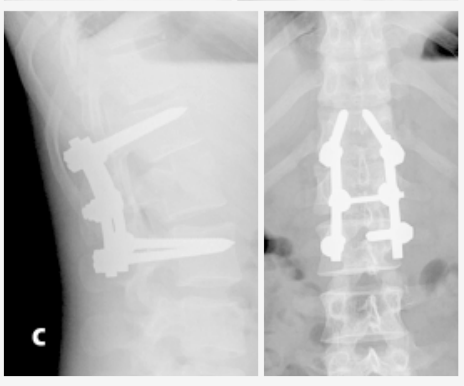

Abb. $2<$ 30-jähriger Patient mit kranialem Berstungsspaltbruch (A3.2.1) von L1: Unfall-CT (a) und konventionelle Unfallröntgenbilder (b); einzeitig kombinierte, dorsoventrale Stabilisierung bisegmental mit thorakoskopischer ventraler Spanfusion monosegmental (c), unveränderte Stellung und Fusion 1 Jahr postoperativ und nach Implantatentfernung (d)
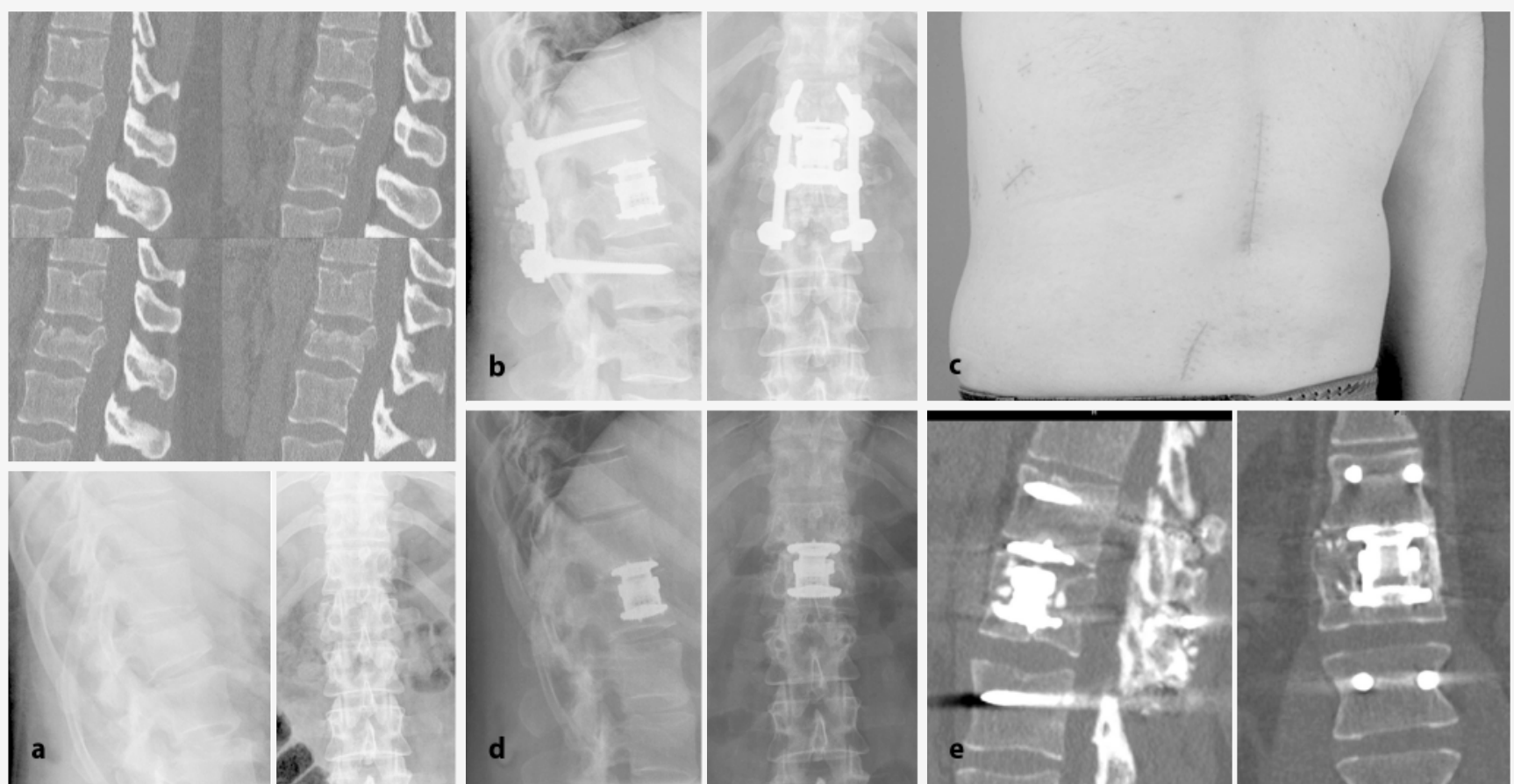

Abb. 3 33-jähriger Patient mit inkomplettem kranialem Berstungsbruch (A3.1.1) von L1 (a). Kombinierte dorsoventrale Operation mit dorsaler Reposition und Stabilisierung mit Fixateur interne bisegmental sowie thorakoskopischem Wirbelkörperersatz monosegmental bei Übergewicht (b). Patient nach 6 Wochen beschwerdefrei und wieder voll berufstätig als Kfz-Mechaniker (c). Ausheilungsergebnis 1 Jahr postoperativ und nach Implantatentfernung (d). Unveränderte Lage des Wirbelkörperersatzimplantates bei interkorporeller knöcherner Fusion in sagittalen und koronaren Rekonstruktionen des Computertomogrammes (e) 
z. T. auch veralteten Operationsmethoden beeinträchtigt.

Verfechter einer operativen Behandlung folgen dagegen der Vermutung, dass ein Zusammenhang zwischen der Dauer einer mechanischen Kompression des Rückenmarks und der Chance einer Rückbildung neurologischer Ausfälle besteht. Eine Reihe von Autoren sprechen sich jedoch aufgrund experimenteller [22] und klinischer Ergebnisse [25, 26, 30, 68, 71, 74] für die operative Dekompression bei neurologischem Defizit aus. Unserer Meinung nach muss man jedem inkomplett Gelähmten heute die Chance auf eine schnellstmögliche Operation geben. Schnellstmöglich bedeutet dabei, dass für den Eingriff immer die optimalen Bedingungen gegeben sein müssen. Der Patient sollte zwar so früh wie möglich operiert, jedoch unter keinen Umständen dadurch gefährdet werden, dass die Behandlung überhastet und durch ein weniger erfahrenes Operationsteam begonnen wird. Auch eine Verlegung in ein geeignetes Zentrum ist nach entsprechender Reposition und Lagerung als Minimalmaßnahme zu erwägen. Als einzige „echte“ Notfallindikation ist die sehr seltene Situation eines zunehmenden neurologischen Defizits, einer schlimmer werdenden Lähmung, zu nennen.

Nicht übersehen werden darf dabei auch die Stabilisierung im Zuge der Dekompression. Dadurch wird eine zusätzliche Schädigung durch sekundäre Verschiebung oder Dislokation zuverlässig vermieden.

\section{Ergebnisse mit Synex}

Erfasst wurden klinische und radiologische Daten der ersten 50 konsekutiven $\mathrm{Pa}$ tienten während des stationären Verlaufes und nach 12 und 18 Monaten. Es handelte sich um 29 Männer und 21 Frauen mit überwiegend frischen, traumatischen Frakturen $(n=36)$ oder veralteten Verletzungen $(n=10)$. 30-mal wurde ein bisegmentaler, 20-mal ein monosegmentaler Wirbelkörperersatz mit Synex durchgeführt. 45 von 50 Patienten wurden 1 Jahr postoperativ und davon 39 nach 1 1/2 Jahren nachuntersucht.

25 Patienten hatten die vorherigen Freizeitaktivitäten wieder aufgenommen, nach 18 Monaten waren es 29 von 39 nachunter- suchten Patienten. 33 von 45 Patienten waren vor der Erstoperation erwerbstätig gewesen, nach 12 Monaten waren knapp die Hälfte, nach 1 1/2 Jahren 2/3 von ihnen wieder im alten Beruf tätig. 25 von 45 Patienten hatten nach 1 Jahr keine oder nur geringe Rückenbeschwerden, nach 18 Monaten waren noch 6 Patienten in ihrer Rückenfunktion eingeschränkt. Drei von 45 Patienten wiesen nach 12 Monaten eine relevante Zugangsmorbidität auf, nach $18 \mathrm{Mo}$ naten waren alle nachuntersuchten Patienten von Seiten des Zuganges weitgehend oder komplett beschwerdefrei.

Der Wirbelsäulenscore, gemessen mit einer visuellen Analogskala, fiel signifikant ab von $87 / 100$ präoperativ auf $68 / 100$ nach 1 Jahr ( $\mathrm{p}<\mathrm{o}, \mathrm{oo1})$. Durch die Operation wurde eine signifikante Korrektur von durchschnittlich $19,2^{\circ}$ erreicht. Nach 12 Monaten verblieb nach einem Korrekturverlust von $2,3^{\circ}$ ein Korrekturgewinn von $16,9^{\circ}$. Eine knöcherne Überbrückung des Synex war bei $83 \%$ der Patienten im konventionellen Röntgenbild nachweisbar.

\section{Ausblick}

Nach Verletzungen der Wirbelsäule und des Rückenmarks waren im Vergleich zu denen aller anderen großen „Organsysteme" die schlechtesten funktionellen Resultate und die niedrigste Wiedereingliederungsrate in das Berufsleben zu beobachten. Diese Feststellung von Johansson et al. 1993 macht den Wert der Suche nach einer optimalen, patientenorientierten Behandlung von Verletzungen der Brustund Lendenwirbelsäule deutlich. Dabei ist neben dem individuellen Wunsch nach möglichst vollständiger Wiederherstellung auch die gesellschaftliche Bedeutung einer beruflichen Rehabilitation der überwiegend jungen Patienten zu berücksichtigen.

Für die kommenden Jahre ist damit zu rechnen, dass die technische Entwicklung der Operationsmethoden fortschreitet und sich überlegene Methoden herauskristallisieren. Die Morbidität des ventralen Eingriffs wird durch die Standardisierung und Verbreitung des endoskopischen Vorgehens unbedeutender werden. Die Dauerfolgen eines dorsalen Eingriffes werden im direkten Vergleich möglicherwei- se überwiegen, wobei es auch hier Bestrebungen zur Minimierung des Operationstraumas durch perkutanes Vorgehen gibt.

Bis heute verfügt kein ventral stabilisierendes Implantat über die Repositionsmöglichkeiten eines dorsal eingebrachten Fixateur interne. Hier sind auch Implantate in der Entwicklung, die einerseits endoskopisch eingebracht werden können und zusätzlich die Möglichkeit bieten sollen, eine Fehlstellung durch den minimal-invasiven Zugang zu reponieren. Das Ziel der Entwicklung wäre, dass auf den dorsalen Eingriff überhaupt verzichtet werden kann.

Last but not least wurden Scores entwickelt und validiert [48], die eine zunehmende Verbreitung finden und für die $\mathrm{Zu}$ kunft auch den Vergleich von Ergebnissen nach unterschiedlichen Behandlungsmethoden ermöglichen werden. Wichtige Hinweise erhoffen sich die beteiligten Kliniken der Arbeitsgemeinschaft „Wirbelsäulenchirurgie" der Deutschen Gesellschaft für Unfallchirurgie von der laufenden Studie „Multicenter II“, die die aktuellen operativen und nichtoperativen Behandlungsmethoden an der gesamten Brust- und Lendenwirbelsäule berücksichtigt [49].

\section{Korrespondierender Autor}

\section{Ao. Univ.-Prof. Dr. C. Knop, M.D.}

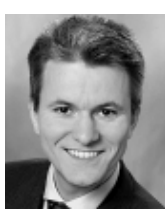

Universitätsklinik für Unfallchirurgie \& Sporttraumatologie, Medizinische Universität Innsbruck,

Anichstraße 35,

6020 Innsbruck, Österreich E-Mail: christian.knop@uibk.ac.at

Interessenkonflikt: Der korrespondierende Autor versichert, dass keine Verbindungen mit einer Firma, deren Produkt in dem Artikel genannt ist, oder einer Firma, die ein Konkurrenzprodukt vertreibt, bestehen.

\section{Literatur}

1. Aebi M, Etter C, Kehl T, Thalgott J (1987) Stabilization of the lower thoracic and lumbar spine with the internal spinal skeletal fixation system. Indications, techniques, and first results of treatment. Spine 12: 544-551

2. Aglietti P, Di Muria GV, Taylor TK et al. (1983) Conservative treatment of thoracic and lumbar vertebral fractures. Ital J Orthop Traumatol 9 [Suppl]: 83105 
3. An HS, Vaccaro A, Cotler JM, Lin S (1991) Low lumbar burst fractures. comparison among body cast, harrington rod, luque rod, and steffee plate. Spine 16 [Suppl]: S440-444

4. Andreychik DA, Alander DH, Senica KM, Stauffer ES (1996) Burst fractures of the second through fifth lumbar vertebrae. J Bone Joint Surg [Am] 78: 11561166

5. Banwart JC, Asher MA, Hassanein RS (1995) lliac crest bone graft harvest donor site morbidity. A statistical evaluation. Spine 20: 1055-1060

6. Been HD (1991) Anterior decompression and stabilization of thoracolumbar burst fractures by the use of the Slot-Zielke device. Spine 16: 70-77

7. Beisse R, Potulski M, Bühren V (1999) Thorakoskopisch gesteuerte ventrale Plattenspondylodese be Frakturen der Brust- und Lendenwirbelsäule. Operat Orthop Traumatol 11: 54-69

8. Beisse R, Potulski M, Temme C, Bühren V (1998) Das endoskopisch kontrollierte Zwerchfellsplitting Ein minimal-invasiver Zugang zur ventralen Versorgung thorakolumbaler Frakturen der Wirbelsäule. Unfallchirurg 101: 619-627

9. Blattert TR, Delling G, Weckbach A (2002) Pedikuloskopisch assistierte transpedikuläre Spongiosaplastik zur interkorporellen Fusion an der lumbalen Wirbelsäule. Unfallchirurg 105: 680-687

10. Blauth M (1998) 1. Grundlagen der Wirbelsäulentraumatologie. In: Tscherne H, Blauth M (Hrsg) Tscherne Unfallchirurgie - Wirbelsäule, 1. Aufl. Springer, Berlin Heidelberg New York Tokio, S 1-59

11. Blauth M, Knop C, Bastian L (1998) 4. Brust- und Lendenwirbelsäule. In: Tscherne H, Blauth M (Hrsg) Tscherne Unfallchirurgie - Wirbelsäule, 1. Aufl. Springer, Berlin Heidelberg New York Tokio, S 241372

12. Blauth M, Knop C, Bastian L, Lobenhoffer P (1997) Neue Entwicklungen in der Chirurgie der verletzten Wirbelsäule. Orthopäde 26: 437-449

13. Boerger TO, Limb D, Dickson RA (2000) Does,canal clearance' affect neurological outcome after thoracolumbar burst fractures? J Bone Joint Surg $[\mathrm{Br}] 82$ 629-635

14. Braun W, Markmiller M, Rüter A (1991) Konservative Therapie von Frakturen der Brust- und Lendenwirbelsäule. Indikation, Behandlungsregime, Ergebnisse. Chirurg 62: 404-408

15. Briem D, Rueger JM, Linhart W (2003) Einheilung autogener Transplantate nach dorsoventraler $\mathrm{In}$ strumentierung instabiler Frakturen der thorakolumbalen Wirbelsäule. Unfallchirurg 106: 195-203

16. Bühren V, Beisse R, Potulski M (1997) Minimal-invasive ventrale Spondylodesen bei Verletzungen der Brust- und Lendenwirbelsäule. Chirurg 68: 10761084

17. Crawford RJ, Askin GN (1994) Fixation of thoracolumbar fractures with the Dick fixator: the influence of transpedicular bone grafting. Eur Spine $\mathrm{J}$ : 45-51

18. Daniaux H (1986) Transpedikuläre Reposition und Spongiosaplastik bei Wirbelkörperbrüchen der unteren Brust- und Lendenwirbelsäule. Unfallchirurg 89: 197-213

19. Day B, Kokan P (1977) Compression fractures of the thoracic and lumbar spine from compensable injuries. Clin Orthop 124: 173-176

20. Denis F, Ruiz H, Searls K (1984) Comparison between square-ended distraction rods and standard round-ended distraction rods in the treatment of thoracolumbar spinal injuries. A statistical analysis. Clin Orthop 189: 162-167

21. Dick W (1987) The "fixateur interne" as a versatile implant for spine surgery. Spine 12: 882-900
22. Dimar JR, Glassman SD, Raque GH et al. (1999) The influence of spinal canal narrowing and timing of decompression on neurologic recovery after spinal cord contusion in a rat model. Spine 24: 16231633

23. Esses SI, Botsford DJ, Wright T et al. (1991) Operative treatment of spinal fractures with the AO internal fixator. Spine 16 [Suppl]: S146-S150

24. Eysel P, Meinig G, Sanner F (1991) Vergleichende Untersuchung unterschiedlicher dorsaler Stabilisierungsverfahren bei frischen Frakturen der Rumpfwirbelsäule. Unfallchirurgie 17: 264-273

25. Fehlings M, Lali HSS, Tator C (2001) The role and timing of decompression in acute spinal cord injury: What do we know? What should we do? Spine 26 [24S]: S101-S110

26. Fehlings M, Tator C (1999) An evidence-based review of decompressive surgery in acute spinal cord injury: rationale, indications, and timing based on experimental and clinical studies. J Neurosurg 91 [Suppl]: 1-11

27. Feil J, Wörsdörfer O (1992) Ventrale Stabilisierung im Bereich der Brust- und Lendenwirbelsäule. Chirurg 63: 856-865

28. Förster R, Storck M, Schäfer JR, Hönig E, Lang G, Liewald F (2002) Thoracoscopy versus thoracotomy: a prospective comparison of trauma and quality of life. Langenbecks Arch Surg 387: 32-36

29. Furrer M, Rechsteiner R, Signer C et al. (1997) Thoracotomy and thoracoscopy: postoperative pulmonary function, pain and chest wall complaints. Eur J Cardiothorac Surg 12: 82-87

30. Gaebler C, Maier R, Kutscha-Lissberg F et al. (1999) Results of spinal cord decompression and thoracolumbar pedicle stabilisation in relation to the time of operation. Spinal Cord 37: 33-39

31. Gertzbein SD (1992) Scoliosis Research Society. Multicenter spine fracture study. Spine 17: 528540

32. Giudicelli R, Thomas P, Lonjon T et al. (1994) Videoassisted minithoracotomy versus muscle-sparing thoracotomy for performing lobectomy. Ann Thorac Surg 58: 712-717

33. Gotzen L, Junge A, Koppelberg T, Stiletto R (1995) Fortschritte in der Fixateur-interne-Stabilisierung von thorakolumbalen Wirbelsäulenverletzungen Unfallchirurg 98: 79-86

34. Gotzen L, Puplat D, Junge A (1992) Indikation, Technik und Ergebnisse monosegmentaler dorsaler Spondylodesen bei Keilkompressionsfrakturen (Grad II) der thorakolumbalen Wirbelsäule. Unfallchirurg 95: 445-454

35. Goulet JA, Senunas LE, De Silva GL, Greenfield ML (1997) Autogenous iliac crest bone graft. Complications and functional assessment. Clin Orthop 339: 76-81

36. Kaltenecker G, Kwasny O, Maier R et al. (1992) Ergebnisse nach konservativ versorgten Wirbelfrakturen am thorakolumbalen Ubergang unter besonderer Berücksichtigung knöcherner Stenosen des Spinalkanals. Unfallchirurg 95: 118-123

37. Kaminski A, Müller EJ, Hankemeier S, Muhr G (2003) Low lumbar spine fracture. Eur J Trauma 29: 23-30

38. Kaneda K, Taneichi H, Abumi K et al. (1997) Anterior decompression and stabilization with the kaneda device for thoracolumbar burst fractures associated with neurological deficits. J Bone Joint Surg [Am] 79 (1): 69-83

39. Karjalainen M, Aho AJ, Katevuo K (1991) Painful spine after stable fractures of the thoracic and lumbar spine. What benefit from the use of extension brace? Ann Chir Gynaecol 80: 45-48
40. Knop C, Blauth M, Bühren V, Arand M et al. (2001) Operative Behandlung von Verletzungen des thorakolumbalen Übergangs - Teil 3: Nachuntersuchung. Unfallchirurg 104: 583-600

41. Knop C, Blauth M, Bühren V et al. (1999) Operative Behandlung von Verletzungen des thorakolumbalen Übergangs - Teil 1: Epidemiologie. Unfallchirurg 102: 924-935

42. Knop C, Blauth M, Bühren V et al. (2000) Operative Behandlung von Verletzungen des thorakolumbalen Übergangs - Teil 2: Operation und röntgenologische Befunde. Unfallchirurg 103: 1032-1047

43. Knop C, Fabian HF, Bastian L, Blauth M (2001) Late results of thoracolumbar fractures after posterior instrumentation and transpedicular bone grafting. Spine 26: 88-99

44. Knop C, Fabian HF, Bastian L et al. (2002) Fate of the transpedicular intervertebral bone graft after posterior stabilisation of thoracolumbar fractures. Eur Spine J 11: 251-257

45. Knop C, Lange U, Bastian L, Blauth M (2000) Threedimensional motion analysis with Synex ${ }^{\mathrm{TM}}-\mathrm{Com}^{-}$ parative biomechanical test series with a new vertebral body replacement for the thoracolumbar spine. Eur Spine J 9: 472-485

46. Knop C, Lange U, Bastian L et al. (2001) Biomechanical compression tests with a new implant for thoracolumbar vertebral body replacement. Eur Spine J 10: 30-37

47. Knop C, Lange U, Blauth M (2004) Der Wirbelkörperersatz mit Synex bei kombinierter dorso-ventraler Behandlung thorakolumbaler Verletzungen. Operat Orthop Traumatol (im Druck)

48. Knop C, Oeser M, Bastian L et al. (2001) Entwicklung und Validierung des VAS Wirbelsäulen-Scores. Unfallchirurg 104: 488-497

49. Knop C, Reinhold M, Schmid R et al. (2004) Internet based multicenter study for thoracolumbar injuries - a new concept and preliminary results. Eur Spine J (eingereicht)

50. Kossmann T, Ertel W, Platz A, Trentz O (1999) Die kombinierte Operation von Frakturen des thorakolumbalen Übergangs mit der Inlay-Span-Technik. Orthopäde 28: 432-440

51. Kostuik JP (1988) Anterior fixation for burst fractures of the thoracic and lumbar spine with or without neurological involvement. Spine 13: 286293

52. Kurz LT, Garfin SR, Booth RE Jr (1989) Harvesting autogenous iliac bone grafts. A review of complications and techniques. Spine 14: 1324-1331

53. Landreneau RJ, Hazelrigg SR, Mack MJ et al. (1993) Postoperative pain related morbidity: video-assisted thoracic surgery versus thoracotomy. Ann Thorac Surg 56: 1285-1289

54. Lange U, Bastian L, Knop C, Blauth M (2002) Einfluß einer kurzstreckigen Spondylodese des thorakolumbalen Übergangs auf die angrenzenden Wirbelgelenke. Eine biomechanische Studie. Unfallchirurg 105: 359-370

55. Lange U, Knop C, Bastian L, Blauth M (2002) Wirbelkörperersatz mit Synex. Ergebnisse einer prospektiven, multizentrischen Dokumentationsserie mit 126 Patienten. Akt Traumatol 32:1-7

56. Leferink VJ, Veldhuis EF, Zimmerman KW et al. (2002) Classificational problems in ligamentary distraction type vertebral fractures: $30 \%$ of all B-type fractures are initially unrecognised. Eur Spine $\mathrm{J} 11$ : 246-250

57. Leferink VJ, Zimmerman KW, Veldhuis EF et al (2001) Thoracolumbar spinal fractures: radiological results of transpedicular fixation combined with transpedicular cancellous bone graft and posterior fusion in 183 patients. Eur Spine J 10: 517-523 
58. Lewis J, McKibbin B (1974) The treatment of unstable fracture-dislocations of the thoraco-lumbar spine accompanied by paraplegia. J Bone Joint Surg [Br] 56: 603-612

59. Lindsey RW, Dick W (1991) The fixateur interne in the reduction and stabilization of thoracolumbar spine fractures in patients with neurologic deficit. Spine 16 [Suppl]: S140-S145

60. Ludolph E, Hierholzer G (1985) Funktionelle Therapie bei primär instabilen Verletzungen der Brustund Lendenwirbelsäule. Indikation und Grenzen. Zentralbl Chir 110: 585-591

61. Magerl F, Aebi M, Gertzbein SD et al. (1994) A comprehensive classification of thoracic and lumbar injuries. Eur Spine J 3: 184-201

62. Magerl F, Engelhardt P (1994) 3. Brust- und Lendenwirbelsäule - Verlaufsformen. In: Witt AN, Rettig H, Schlegel KF (Hrsg) Orthopädie in Praxis und Klinik, Spezielle Orthopädie (Wirbelsäule - Thorax - Becken), Bd 5 (2), 2. Aufl. Thieme, Stuttgart New York, 3.82-3.132

63. Mayer H, Schaaf D, Kudernatsch M (1992) Der Einsatz des Fixateur interne bei Verletzungen der Brust- und Lendenwirbelsäule. Chirurg 63: 944 949

64. McAfee PC, Yuan HA, Fredrickson BE, Lubicky JP (1983) The value of computed tomography in thoracolumbar fractures. an analysis of one hundred consecutive cases and a new classification. J Bone Joint Surg [Am] 65: 461-473

65. McAfee PC, Yuan HA, Lasda NA (1982) The unstable burst fracture. Spine 7: 365-373

66. Mumford J, Weinstein JN, Spratt KF, Goel VK (1993) Thoracolumbar burst fractures. the clinical efficacy and outcome of nonoperative management. Spine 18: 955-970

67. Okuyama K, Abe E, Chiba M, Ishikawa N, Sato K (1996) Outcome of anterior decompression and stabilization for thoracolumbar unstable burst fractures in the absence of neurologic deficits. Spine 21: 620-625

68. Papadopoulos SM, Selden NR, Quint DJ et al. (2002) Immediate spinal cord decompression for cervical spinal cord injury: feasibility and outcome. J Trauma 52: 323-332

69. Passlick B, Born C, Mandelkow H et al. (2001) Langzeitbeschwerden nach minimal-invasiven thoraxchirurgischen Operationen und nach Thoracotomie. Chirurg 72: 934-938

70. Pickel H, Hofmeister M, Mückley T, Bühren V (1997) Schicksal der transpedikulären Spongiosaplastik nach Frakturversorgung. Hefte Unfallchirurg 268: 251

71. Ransohoff J, Benjamin V, Flamm ES (1982) Early surgical management of acute spinal cord injury. J Am Paraplegia Soc 5: 19-21

72. Reinhold M, Knop C, Lange U et al. (2003) Nichtoperative Behandlung von Verletzungen der thorakolumbalen Wirbelsäule - Klinische Spätergebnisse nach 16 Jahren. Unfallchirurg 106: 566-576

73. Resch H, Rabl M, Klampfer H et al. (2000) Operative vs. konservative Behandlung von Frakturen des thorakolumbalen Übergangs. Unfallchirurg 103: 281-288

74. Rosenfeld JF, Vaccaro AR, Albert TJ et al. (1998) The benefits of early decompression in cervical spinal cord injury. Am J Orthop 27: 23-28

75. Sasso RC, Cotler HB (1993) Posterior instrumentation and fusion for unstable fractures and fracturedislocations of the thoracic and lumbar spine. A comparative study of three fixation devices in 70 patients. Spine 18: $450-460$
76. Sasso RC, Williams JI, Dimasi N, Meyer-PR J (1998) Postoperative drains at the donor sites of iliaccrest bone grafts. A prospective, randomized study of morbidity at the donor site in patients who had a traumatic injury of the spine. J Bone Joint Surg [Am] 80: 631-635

77. Sawin PD, Traynelis VC, Menezes AH (1998) A comparative analysis of fusion rates and donor-site morbidity for autogeneic rib and iliac crest bone grafts in posterior cervical fusions. J Neurosurg 88: 255-265

78. Soreff J, Axdorph G, Bylund P et al. (1982) Treatment of patients with unstable fractures of the thoracic and lumbar spine: a follow-up study of surgical and conservative treatment. Acta Orthop Scand 53: 369-381

79. Stürmer KM, Koeser K, Schax M, Hanke J (1994) Ergebnisse der operativen Behandlung instabiler Frakturen der Brust- und Lendenwirbelsäule. Akt Probl Chir Orthop 43: 67-81

80. Summers BN, Eisenstein SM (1989) Donor site pain from the ilium. A complication of lumbar spine fusion. J Bone Joint Surg [Br] 71: 677-680

81. Tasdemiroglu E, Tibbs PA (1995) Long-term followup results of thoracolumbar fractures after posterior instrumentation. Spine 20: 1704-1708

82. Trojan E (1972) Langfristige Ergebnisse von 200 Wirbelbrüchen der Brust-/Lendenwirbelsäule ohne Lähmung. Unfallmed Berufskrankh 66: 122134

83. Tropiano P, Huang RC, Louis CA et al. (2003) Functional and radiographic outcome of thoracolumbar and lumbar burst fractures managed by closed orthopaedic reduction and casting. Spine 28 : 2459-2465

84. von Gumppenberg S, Vieweg J, Claudi B, Harms $J$ (1991) Die primäre Versorgung der frischen Verletzungen von Brust- und Lendenwirbelsäule. Akt Traumatol 21: 265-273

85. Waelchli B, Heini P, Berlemann U (2001) Korrekturverlust nach dorsaler Stabilisierung von Berstungsfrakturen des thorakolumbalen Übergangs - Die Rolle der transpedikulären Spongiosaplastik. Unfallchirurg 104: 742-747

86. Wagner W, Stolper P (1898) Die Verletzungen der Wirbelsäule und des Rückenmarks. Bergmann E v, Bruns P v (Hrsg). Enke, Stuttgart

87. Weckbach A, Vogel S (1997) Einfluß der transpedikulären intercorporellen Spongiosaplastik auf den Korrekturverlust nach alleiniger dorsaler Instrumentierung thoracolumbaler Wirbelsäulenverletzungen. Hefte Unfallchirurg 268: 205-208

88. Winkler H, Fischer M, Kessler T et al. (1999) Korrekturverlust und Einheilungsverhalten transpedikulärer Spongiosaplastiken bei der Behandlung thorakolumbaler Wirbelfrakturen. Trauma Berufskrankh 1: 294-301

89. Wippermann BW, Schratt HE, Steeg S, Tscherne H (1997) Komplikationen der Spongiosaentnahme am Beckenkamm. Eine retrospektive Analyse von 1191 Fällen. Chirurg 68: 1286-1291

90. Young MH (1973) Long-term consequences of stable fractures of the thoracic and lumbar vertebral bodies. J Bone Joint Surg [Br] 55: 295-300

91. Younger EM, Chapman MW (1989) Morbidity at bone graft donor sites. J Orthop Trauma 3: 192195

92. Yuan HA, Mann KA, Found EM, Helbig TE et al. (1988) Early clinical experience with the Syracuse I-Plate: an anterior spinal fixation device. Spine 13: 278-285 